\title{
High-dynamic strengthening of cementitious materials subjected to uniaxial compression
}

\author{
E. Binder \& H.A. Mang \\ Institute for Mechanics of Materials and Structures, TU Wien —Vienna University of Technology, \\ Vienna, Austria \\ College of Civil Engineering, Tongji University, Shanghai, China \\ Y. Yuan \\ College of Civil Engineering, Tongji University, Shanghai, China \\ B. Pichler \\ Institute for Mechanics of Materials and Structures, TU Wien -Vienna University of Technology, \\ Vienna, Austria
}

\begin{abstract}
Infrastructures made of reinforced concrete such as e.g. tunnels and bridges, must withstand exceptional loads, caused by earthquakes, car impacts, accidential blast loads, etc. The latter two belong to the high-dynamic loading regime where strain rates are typically larger than $1 / \mathrm{s}$. In this regime, the strength of concrete specimens increases significantly with increasing loading rate. The reason for this strengthing effect is still debated. This provides the motivation for the present contribution which is devoted to the analysis of high-dynamic compression tests on cement pastes, mortars, and concretes. An elasto-brittle model for high-dynamic strengthening is employed, which was proposed by Fischer et al. (2014) and extended towards consideration of the scatter of the quasi-static strength values by Binder et al. (2017). The aim of the present contribution is twofold. At first, the quantitative assessment of the predictive capabilities of the described model is extended towards consideration of experimental data from Hao \& Hao (2013). Secondly, the question is tackled how to represent high-dynamic strength values of different materials (cement pastes, mortars, and concretes) in one diagram, such that a meaningful direct comparison becomes possible. The latter goal is achieved by comparing two independent measures of the increase of the compressive strain during the failure process of a specimen: the first one refers to the measured Dynamic strength Increase Factor (DIF), the quasi-static strength, and Young's modulus and the second one to the the strain rate, the characteristic crack propagation length, and the crack propagation speed.
\end{abstract}

\section{INTRODUCTION}

Current safety standards demand that tunnels and bridges must withstand exceptional load cases such as impact and blast loads. The latter may result from traffic accidents, e.g. from cars crashing into a tunnel lining, or from the detonation of so-called Improvised Explosive Devices (Ngo, Mendis, Gupta, \& Ramsay 2007, Solz \& Ruiz-Ripoll 2016). This provides the motivation to investigate concrete subjected to high-dynamic loading.

It was shown in many high-dynamic experiments that the strength of cementitious materials increases with increasing loading rate, see, e.g., (Bischoff and Perry 1991, Gary and Bailly 1998, Grote et al. 2001, Tedesco and Ross 1998, Xu et al. 2012, Zhang et al. 2009). This strengthening is significant in the high dynamic testing regime, where strain rates are typically larger than $1 / \mathrm{s}$.

The available experimental database has resulted in several modeling attempts. The CEBrecommendation (1993) as well as the models of Tedesco and Ross (1998) and of Grote et al. (2001) provide empirical formulae for the high-dynamic compressive strength of cementitious materials. Mihashi and Wittmann (1980) as well as Bažant et al. (2000) have developed models based on the assumption that micro-cracking depends on the strain rate. Cotsovos and Pavlovic (2008), Li and Meng (2003), and Gary and Bailly (1998) have attributed the strength increase with increasing strain rate to inertial confinement which is a structural effect. Fischer et al. (2014) related the dynamic strength increase to the quasi-static strength and to 
the duration of the failure process, lasting from the start of crack propagation to the final disintegration of the tested specimen. Thereby, it was considered that loading of the specimen increases during the evolution of the failure process. The load level reached at the end of the failure process is the highdynamic strength of the tested specimen.

The aim of the present contribution is twofold. At first, the exemplary validation of the model by Fischer et al. (2014) is extended towards consideration of experimental data from Hao \& Hao (2013). Secondly, the question is tackled how to represent high-dynamic strength values of different materials (cement pastes, mortars, and concretes) in one diagram, such that a reasonable direct comparison becomes possible.

The present paper is structured as follows. Section 2 contains a brief overview over the necessary prerequisites for the present analysis. Section 3 is devoted to the assessment of the predictive capabilities of the investigated DIF model. Section 4 establishes the sought direct compability of high-dynamic strength values of different cementitious materials. Section 5 contains a summary and conclusions. Throughout the entire manuscript, a positive sign in mathematical expressions relates to compression.

\section{ELASTO-BRITTLE PREDICTION OF HIGH-DYNAMIC STRENGTH, BASED ON QUASI-STATIC STRENGTH DATA}

In this section, the developments of Fischer et al. (2014), Pichler et al. (2014), and Binder et al. (2017) are briefly summarized. The respective elasto-brittle model for the dynamic increase factor (DIF) of the compressive strength of cementitious materials was derived, based on the following considerations:

- Cracking is considered to start also under highdynamic loading, once the quasi-static strength is reached.

- The failure process of the specimen starts at the onset of cracking and ends once the first crack splits the specimen such that it disintegrates into pieces.

- During the failure process, it is possible to further increase the loading.

- Cracks are considered to propagate along nanoscopic interfaces at a speed, estimated to be equal to the shear wave velocity $v_{s}$. Notably, $v_{s}$ is only by a few percent greater than the Rayleigh wave speed, and the latter is frequently considered to be a good estimate of the crack propagation velocity (Freund 1998).

- The shear wave velocity can be quantified, based on known values of the shear modulus $G$ and the mass density $\rho$, as $v_{s}=\sqrt{G / \rho}$. The shear modulus, in turn, is related to the Young's modulus $E$ and Poisson's ratio $v$ as $G=E /[2(1+v)]$.

- The loading reached at the end of the failure process, i.e. immediately before disintegration of the specimen, is equal to its high-dynamic strength.

This line of arguments has led to the follwoing closed-form expression of the DIF, defined as the dynamic-to-static strength ratio, see Fischer et al. Fischer et al. (2014).

$D I F=\frac{f_{d y n}}{f_{\text {stat }}}=1+\frac{E \dot{\varepsilon}}{f_{\text {stat }}} \frac{l_{c}}{v_{s}}$.

In Eq. (1), $f_{d y n}, f_{\text {stat }}, \dot{\varepsilon}$, and $l_{c}$ stand for the dynamic strength, the quasi-static strength, the strain rate, and the relevant crack propagation length. In the context of elasto-brittle modeling, the products $E \dot{\varepsilon}$ and $l_{c} / v_{s}$ can be interpreted as the elastic stress rate and the duration of the failure process, respectively.

\subsection{Uncertainty regarding the position of crack nucleation}

The crack length, $l_{c}$, is equal to the distance along which a crack must propagate in order to split the specimen. Therefore, $l_{c}$ depends on the geometrical properties of the tested specimen and on the (a priori unknown) position at which the first crack nucleates. Lack of knowledge of the latter is a source of uncertainty. Still, considering that axial splitting is the typical failure mode under uniaxial compression, $l_{c}$ is bounded as follows:

- The smallest value of $l_{c}$ is obtained, if the crack nucleates in the middle of the specimen, such that both crack tips have to propagate along half of the specimen height. Therefore, the lower bound is given as $l_{c}=h / 2$.

- The largest value of $l_{c}$ is obtained if the crack nucleates right at one of the interfaces between the specimen and the adjacent load application system, such that the crack tip has to propagate along the total height of the specimen. Therefore, the upper bound is given as $l_{c}=h$.

\subsection{Uncertainty regarding the quasi-static strength}

As regards quantification of the statistical scatter of the quasi-static strength, see $f_{\text {stat }}$ in Eq. (1), the estimation of statistical quantiles is desirable. Unfortunately, this is often impossible, because experimentalists frequently only communicate the mean value of the quasi-static strenght. As a remedy, 
Binder et al. (2017) combined the statistical scatter considered by Eurocode 2 (EN 1992-1-1 (2011)), which refers to a Gaussian distribution, with quantiles of a corresponding lognormal distribution.

Eurocode 2 defines the characteristic strength for ultimate limit state design as the $5 \%$-quantile of the strength distribution. Concerning the compressive strength of concrete, the 5\%-quantile is set equal to a value which is by $8 \mathrm{MPa}$ smaller than the mean strength determined by laboratory testing. Considering a Gaussian distribution, this approach is related to a standard deviation of the uniaxial compressive strength amounting to $\hat{s}=4.865 \mathrm{MPa}$.

A lognormal distribution appears to be more appropriate to describe the statistical properties of the compressive strength, which is a strictly positive quantity. In order to convert the standard deviation from Eurocode 2 to the one from the envisioned lognormal distribution, it is proposed to set the 5\%-quantile of the Gaussian distribution equal to the $5 \%$-quantile of the lognormal distribution. Denoting the value of the mean strength from the experiments as $\hat{f}$ and the standard deviation of the Eurocode as $\hat{s}$, the proposed approach from Binder et al. (2017) yields the sought standard deviation of the natural logarithm of the quasistatic compressive strength distribution as,

$$
\bar{s}=u_{5 \%}+\sqrt{\left(u_{5 \%}\right)^{2}-2 \ln \left(1+\frac{\hat{s} \cdot u_{5 \%}}{\hat{f}}\right)},
$$

where $u_{5^{\circ}}=-1.645$ is the value that cuts an area of $5 \%$ of the standardized normal distribution, exhibiting a vanishing mean value.

Using the standard deviation of the natural logarithm of the quasi-static compressive strength distribution according to Eq. (2), any p-quantile of the lognormal distribution can be calculated according to the following standard relation:

$$
f_{p}=\exp \left(\ln (\hat{f})+\bar{s} \cdot u_{p}\right) .
$$

In the following, the $5 \%$ and $95 \%$-quantiles of the lognormal distribution will be used as upper and lower bounds of the quasi-static compressive strength. To this end, $p$ in Eq. (3) is set equal to 5\% and to $95 \%$, respectively, noting that $u_{95 \%}$ amounts to $+1: 645$.

\section{QUANTITATIVE ASSESSMENT OF THE INVESTIGATED DIF MODEL}

The investigated DIF model is applied to cement pastes, mortars, and concretes. In order to assess the predictive capabilities of the model, experimental material was selected that provides the necessary input for the model, such that no parameter needs to be fitted.

\subsection{Cement paste tested by Fischer et al. (2014)}

Fischer et al. (2014) crushed cement paste cylinders under quasi-static conditions and with a Split Hopkinson Pressure Bar (SHPB), see Table 1 for the experimental data. Corresponding model predictions agree qualitatively and quantitatively very well with the experimental data, see Fig. 1.

\subsection{Mortar tested by Zhang et al. (2009)}

Zhang et al. (2009) crushed mortar cylinders with two different sizes: one group exhibited $h_{1}=18 \mathrm{~mm}$ and $\varnothing_{1}=37 \mathrm{~mm}$, the other one $h_{2}=25 \mathrm{~mm}$ and $\varnothing_{2}=50 \mathrm{~mm}$, see Table 2 for the experimental data. Corresponding model predictions agree both qualitatively and quantitatively very well with

Table 1. Experimental data regarding high-dynamic strength testing by Fischer et al. (2014) from cement paste cylinders: $f_{\text {stat }}=48.16 \mathrm{MPa} ; E=14.24 \mathrm{GPa} ; G=5.53 \mathrm{GPa}$; $\varnothing=10 \mathrm{~mm} ; h=6.6 \mathrm{~mm} ; \rho=1593 \mathrm{~kg} / \mathrm{m}^{3}$.

\begin{tabular}{cccc}
\hline$f_{d y n}[\mathrm{MPa}]$ & $\dot{\varepsilon}\left[\mathrm{s}^{-1}\right]$ & $f_{d y n}[\mathrm{MPa}]$ & $\dot{\varepsilon}\left[\mathrm{s}^{-1}\right]$ \\
\hline 74.15 & 700 & 132.73 & 1900 \\
42.01 & 200 & 164.39 & 2100 \\
74.41 & 500 & 156.36 & 2100 \\
48.60 & 500 & 133.73 & 2100 \\
65.40 & 500 & 152.64 & 2100 \\
114.26 & 5000 & 143.78 & 1900 \\
\hline
\end{tabular}

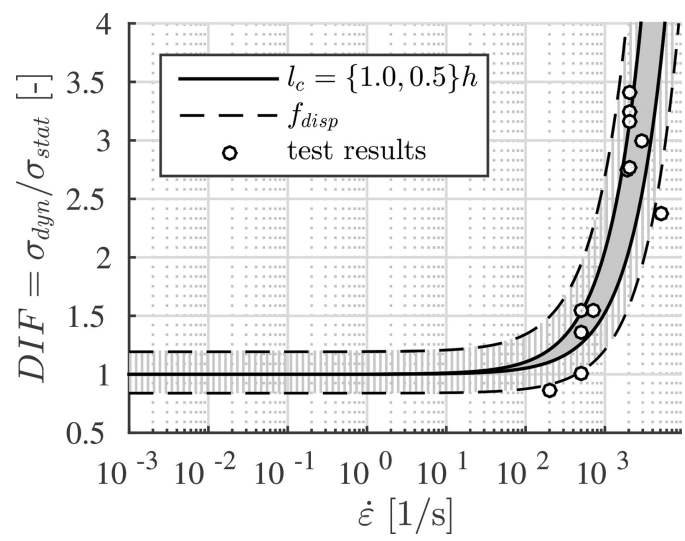

Figure 1. Comparison of increase of the modelpredicted high-dynamic strength according to Eq. (1) with measured dynamic strength increase factors from Fischer et al. (2014), see Table 1. 
the experimental data, see Fig. 2 as well as Wang et al. (2017).

\subsection{Concrete tested by Hao and Hao (2013)}

In this contribution, exemplary model validation is extended to high-dynamic strength tests performed by Hao \& Hao (2013). They tested plain concrete cylinders with a diameter of $50 \mathrm{~mm}$ and a height of $80 \mathrm{~mm}$, see Table 3 for the experimental data. Because they have neither reported the shear modulus nor Poisson's ratio of the tested concrete, the latter is estimated as the standard value of concrete: $v=0.2$. Corresponding model predictions agree both qualitatively and quantitatively very well with the experimental data, see Fig. 3.

Table 2. Experimental data regarding high-dynamic strength testing by Zhang et al. (2009) from mortar cylinders: $f_{\text {stat }}=51 \mathrm{MPa} ; E=23.1 \mathrm{GPa} ; v=0.19 ; \rho=2116 \mathrm{~kg} /$ $\mathrm{m}^{3}$; (a) $h=18 \mathrm{~mm} ; \varnothing=37 \mathrm{~mm}$, and (b) $h=25 \mathrm{~mm}$; $\varnothing=50 \mathrm{~mm}$.

\begin{tabular}{lccc}
\hline$f_{d y n}[\mathrm{MPa}]$ & $\dot{\varepsilon}\left[\mathrm{s}^{-1}\right]$ & $f_{d y n}[\mathrm{MPa}]$ & $\dot{\varepsilon}\left[\mathrm{s}^{-1}\right]$ \\
\hline 70.56 & 49 & 86.57 & 205 \\
73.82 & 71 & 90.44 & 295 \\
74.75 & 85 & 96.52 & 338 \\
75.11 & 131 & 98.16 & 346 \\
75.69 & 153 & 99.45 & 323 \\
79.67 & 184 & 101.67 & 330 \\
81.66 & 220 & 109.04 & 453 \\
64.45 & 37 & 79.14 & 126 \\
71.22 & 69 & 82.03 & 165 \\
71.47 & 76 & 88.80 & 192 \\
75.02 & 86 & 93.34 & 165 \\
72.13 & 89 & 93.75 & 229 \\
76.01 & 126 & 98.62 & 261 \\
77.00 & 153 & & \\
\hline
\end{tabular}

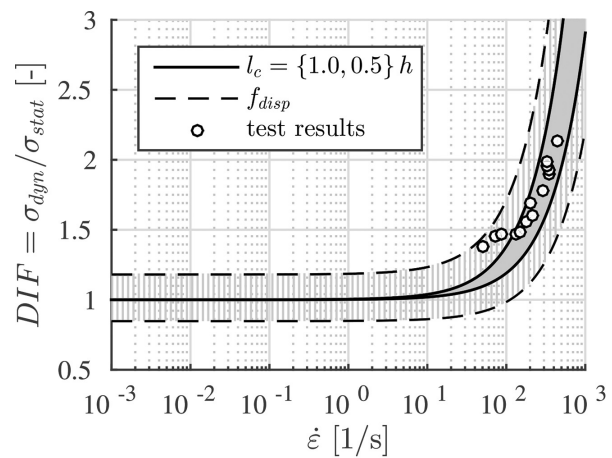

(a)
Table 3. Experimental data regarding high-dynamic strength testing by Hao \& Hao (2013) from concrete cylinders: $f_{\text {stat }}=35.5 \mathrm{MPa} ; E=28.29 \mathrm{GPa} ; \varnothing=75 \mathrm{~mm}$; $h=37.5 \mathrm{~mm} ; \rho=2201 \mathrm{~kg} / \mathrm{m}^{3}$.

\begin{tabular}{lllr}
\hline$f_{d y n}[\mathrm{MPa}]$ & $\dot{\varepsilon}\left[\mathrm{s}^{-1}\right]$ & $f_{d y n}[\mathrm{MPa}]$ & $\dot{\varepsilon}\left[\mathrm{s}^{-1}\right]$ \\
\hline 36.57 & $1.3110^{-4}$ & 68.52 & 99.4 \\
34.44 & $1.3510^{-4}$ & 58.93 & 103.8 \\
53.25 & 65.4 & 63.55 & 131.3 \\
45.09 & 70.5 & 64.61 & 135.6 \\
53.96 & 79.1 & 62.84 & 141.9 \\
46.51 & 79.2 & 90.53 & 163.6 \\
57.16 & 90.2 & 81.65 & 177.3 \\
65.68 & 98.7 & 81.65 & 175.4 \\
61.77 & 99.1 & & \\
\hline
\end{tabular}

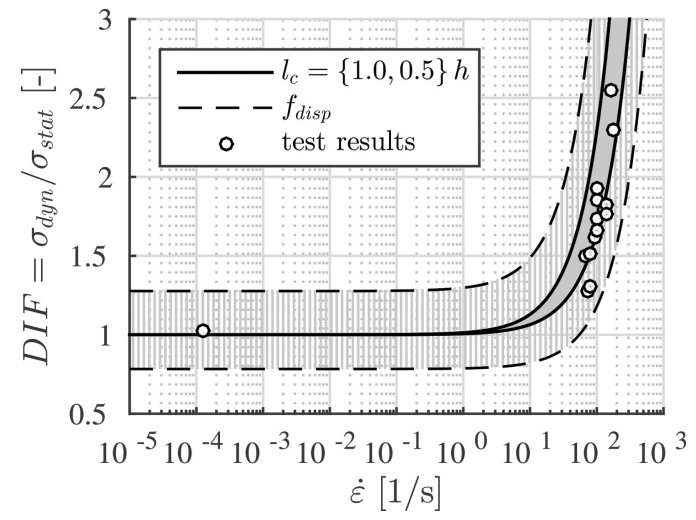

Figure 3. Comparison of increase of the modelpredicted highdynamic strength according to Eq. (1) with measured dynamic strength increase factors from Hao \& Hao (2013) see Table 3.

Figure 2. Comparison of increase of the model-predicted high-dynamic strength according to Eq. (1) with measured dynamic strength increase factors from Zhang et al. (2009): (a) $h=18 \mathrm{~mm}$, and (b) $h=25 \mathrm{~mm}$, see Table 2. 


\section{COMPARISON OF HIGH-DYNAMIC STRENGTH VALUES OF DIFFERENT CEMENTITIOUS MATERIALS}

The analyzed cement pastes, mortars, and concretes are based on different elastic stiffnesses, quasi-static strengths, and specimen dimensions. This renders a direct comparison of the different test results a challenging task. In order to compare data from all analyzed high-dynamic testing series in one diagram, Eq. (1) is rearranged as

$$
(D I F-1) \frac{f_{s t a t}}{E}=\frac{\dot{\varepsilon} l_{c}}{v_{s}} .
$$

The left-hand-side of Eq. (4) can be interpreted as the increase of dynamic strain during the failure process. It is based on the measured DIF values, the quasi-static strength, and on Young's modulus. The right-hand-side of Eq. (4) represents the increase of the dynamic strain, predicted by means of the strain rate, the crack propagation length, and the crack propagation speed.

When it comes to displaying the experimental data summarized in Tables 1, 2, and 3 according to Eq. (4), each one of the high dynamic compression tests is represented by two points: one obtained for $l_{c}=h / 2$, the other one for $l_{c}=h$, see Fig. 4 . All of these data points form a quite dense data cloud. This underlines that Eq. (4) is indeed well suited to compare high-dynamic strength data referring to different materials.

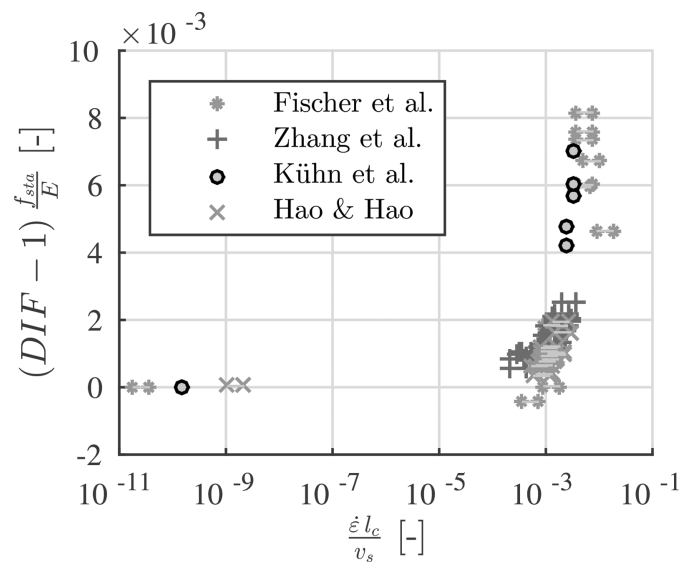

Figure 4. Dimensionless diagram relating two different expressions for the increase of strains during the failure process, see Eq. (4), thus allowing for a direct comparison of high-dynamic strength values of different materials: strength values from high-dynamic testing, taken from Tables 1-3.

\section{SUMMARY AND CONCLUSIONS}

The model used in the present contribution considers that the duration of a compressive strength tests consists of (i) the period of time until crack nucleation and (ii) the duration of the failure process. In the quasi-static regime, the latter is negligibly short compared to the former period of time. In the high-dynamic regime, however, both contributions are of the same order of magnitude.

The good qualitative and quantitative agreement between model predictions and independent experimental data corroborates the essential modeling assumptions that (i) cracking starts, independent of the loading rate, once the quasi-static strength is reached, (ii) cracks propagate approximately at a speed which can be estimated on the basis of the shear wave speed, and (iii) the maximum load of a specimen is reached, once the first crack has propagated such that it splits the specimen.

It is concluded that the high-dynamic strengthening effect of cementitous materials is directly proportional to the duration of the failure process. This was exploited in the context of developing a strategy that allows for a direct comparison of high-dynamic strength tests carried out on different cementitious materials. It is based on the comparison of two independent modes of quantifying the increase of the strain during the failure process.

As for bridges and tunnels, it is concluded that concrete will be damaged if the quasi-static strength is reached or exceeded, no matter how fast the loading is applied. Therefore, infrastructural facilities must be inspected very carefully, even after non-catastrophic high-dynamic loading events.

\section{ACKNOWLEDGMENT}

Financial support by the Austrian Science Fund (FWF), provided within project P 281 31-N32 "Bridging the Gap by Means of Multiscale Structural Analyses", is gratefully acknowledged.

\section{REFERENCES}

Bažant, Z., F. Caner, M. Adley, \& S. Akers (2000). Fracturing rate effect and creep in microplane model for dynamics. Journal of Engineering Mechanics 126, 962-970.

Binder, E., H. Mang, Y. Yuan, \& B. Pichler (2017). Impact and blast loads in tunneling: does the strength of concrete indeed increase with the loading rate? In Proceedings of the IV International Confrence on Computational Methodes in Tunneling and Subsurface Engineering, pp. 773-780. Univercity Innsbruck. 
Bischoff, P. \& S. Perry (1991). Compressive behaviour of concrete at high strain rates. Materials and Structures 24(6), 425-450.

Comite Euro-International du Beton (1993). CEB-FIP Model Code.

Cotsovos, D. \& M. Pavlovic (2008). Numerical investigation of concrete subjected to compressive impact loading. part 1: A fundamental explanation for the apparent strength gain at high loading rates. Computers and Structures 86, 145-163.

European Committee for Standardization (2011). Eurocode 2: Design of concrete structures - part 1-1: General rules and rules for buildings.

Fischer, I., B. Pichler, E. Lach, C. Terner, E. Barraud, \& F. Britz (2014). Compressive strength of cement paste as a function of loading rate: Experiments and engineering mechanics analysis. Cement and Concrete Research 13(58), 186-200.

Freund, L. B. (1998). Dynamic fracture mechanics. Cambridge University Press.

Gary, G. \& P. Bailly (1998). Behaviour of quasi-brittle material at high strain rate. experiment and modelling. European Journal of Mechanics-A/Solids 17(3), 403-420.

Grote, D. L., S. W. Park, \& M. Zhou (2001). Dynamic behavior of concrete at high strain rates and pressure: I. experimental characterization. International Impact Engineering 25(9), 869-886.

Hao, Y. \& H. Hao (2013). Dynamic compressive behaviour of spiral steel fibre reinforced concrete in split hopkinson pressure bar tests. Construction and Building Materials 48, 521-532.

Li, Q. \& H. Meng (2003). About the dynamic strength enhancement of concrete-like materials ina slpit hopkinson pressure bar test. Solids and Structures 40, 343-360.

Mihashi, H. \& F.Wittmann (1980). Stochastic approach to study the influence of rate of loading on strength of concrete. Heron 25, 54.

Ngo, T., P. Mendis, A. Gupta, \& J. Ramsay (2007). Blast loading and blast effects on structures - an overview. Structural Engineering 7, 76-91.

Pichler, B., I. Fischer, E. Lach, C. Terner, E. Barraud, \& F. Britz (2014). The influence of loading rate on the compressive strength of cementitious materials: experiemnts and "separation of time scales" -based analysis. Conference Paper 13(58), 0.

Solz, A. \& M. Ruiz-Ripoll (2016). Experimental and computational characterization of dynamic loading and structural restistance of tunnels in blast scenarios. Fire Technology 52, 1595-1618.

Tedesco, J. \& C. Ross (1998). Strain-rate-dependent constitutive equations for concrete. Journal of Pressure Vessel Technology 120(4), 398-405.

Wang, H., E. Binder, H. Mang, Y. Yuan, \& B. Pichler (2017). Exeptional load cases for tunnel linings. Manuscript in preparation.

Xu, Z., H. Hao, \& H. Li (2012). Experimental study of dynamic compressive properties of fibre reinforced concrete material with different fibres. Materials \& Design 33, 42-55.

Zhang, M., H. Wu, Q. Li, \& F. Huang (2009). Further investigation on the dynamic compressive strength enhancement of concrete-like materials based on split hopkinson pressure bar tests. part i: Experiments. International Journal of Impact Engineering 36(12), 1327-1334. 